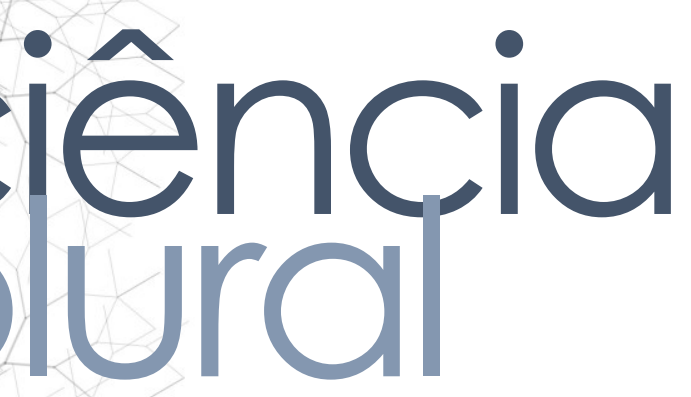

\title{
AV ALIAÇÃO DA AMPLITUDE DE ABERTURA BUCAL EM PACIENTES COM DISFUNÇÃO TEMPOROMANDIBULAR APÓS TRATAMENTO COM TERAPIA MANUAL
}

Evaluation of mouth opening amplitude in patients with temporomandibular disorder after treatment with manual therapy

Evaluación de la amplitud de apertura de la boca en pacientes con transtorno temporomandibular después del tratamiento con terapia manual

Cecília Santos Galvão • Cirurgiã-Dentista • Especializanda em Prótese Dentária • Mestranda em Ciências Odontológicas pela Universidade Federal do Rio Grande do Norte• E-mail: ceciliasgalvao@hotmail.com

Gustavo Augusto Seabra Barbosa • Professor Adjunto da Universidade Federal do Rio Grande do Norte • Coordenador da Base de Pesquisa "Grupos de Estudos e Pesquisas em Reabilitação Oral - GEPRO" • E-mail: gustavoaseabra@hotmail.com Erika Oliveira de Almeida • Professora Adjunto do Departamento de Odontologia da Universidade Federal do Rio Grande do Norte • Doutora em Prótese Dentária E-mail: erika.almeida.protese@gmail.com

Autora responsável pela correspondência:

Cecília Santos Galvão• E-mail: ceciliasgalvao@hotmail.com 


\section{RESUMO}

Introdução: A disfunção temporomandibular, distúrbio que acomete as estruturas ósseas, musculares e articulares da região orofacial tem sido cada vez mais recorrente. Esse distúrbio causa dores, limitação de movimento e alteração na qualidade de vida dos pacientes acometidos com a doença. Se fazem necessárias medidas de controle e tratamento dessa doença de prevalência crescente. Objetivo: Avaliar a amplitude de abertura bucal em pacientes portadores de disfunção temporomandibular, submetidos ao tratamento com fisioterapia após 1 e 3 meses de tratamento. Metodologia: Avaliouse a amplitude de abertura bucal de 25 pacientes submetidos ao tratamento com fisioterapia. Todos os pacientes foram diagnosticados com disfunção de acordo com o eixo 1 do "Research Diagnostic Criteria for Temporomandibular Disorders". Os dados colhidos foram avaliados através do programa SPSS e foi realizado o teste Wilcoxon, com nível de confiança de 95\%. Resultados: Para pacientes que apresentavam comprometimento da amplitude de abertura máxima sem auxílio, a fisioterapia possibilitou melhora significativa do ganho de amplitude no tempo inicial e após um mês de terapia $(p=0,002)$, mantendo a amplitude até o terceiro mês, sem apresentar ganho significativo. Conclusões: Para a variável amplitude de abertura bucal, a fisioterapia se mostra como uma boa alternativa de tratamento, alcançando resultados satisfatórios para o ganho de amplitude e melhoria do quadro sintomático do paciente.

Palavras-Chave: Síndrome da Disfunção da Articulação Temporomandibular; Articulação temporomandibular; Fisioterapia; Amplitude de Movimento Articular.

\section{ABSTRACT}

Introduction: Temporomandibular disorder, a disorder that affects bone, muscle and joint structures in the orofacial region has been increasingly recurrent. This disorder causes pain, movement limitation and changes in the quality of life of patients affected by the disease. Control measures and treatment of the disease of increasing prevalence are necessary. Objective: To evaluate the range of mouth opening in patients undergoing treatment with physiotherapy after 1 and 3 months of treatment. Methodology: It was the amplitude of mouth opening in 25 patients submitted to treatment with physiotherapy. All patients were diagnosed with disorder according to axis 1 of the Research Diagnostic Criteria for Temporomandibular Disorders. The collected data were evaluated using the SPSS program and the Wilcoxon test was performed, with a 95\% confidence level. Results: For patients who presented impairment of the maximum opening amplitude without assistance, physiotherapy enabled a significant improvement in amplitude gain in the initial time and after one month of therapy ( $p=0.002)$, maintaining the amplitude until the third month, without showing any significant gain. Conclusions: For the variable mouth opening mplitude, physiotherapy is shown to be a good treatment alternative, achieving atisfactory results for gaining amplitude and improving the patient's symptomatic ndition.

words: Temporomandibular Joint Dysfunction Syndrome; Temporomandibular t; Physical Therapy Specialty; Range of Motion, Articular. 


\section{ciênciána
piural}

\section{RESUMEN}

Introducción: El trastorno temporomandibular, un trastorno que afecta las estructuras óseas, musculares y articulares en la región orofacial, ha sido cada vez más recurrente. Este trastorno causa dolor, limitación de movimiento y cambios en la calidad de vida de los pacientes afectados por la enfermedad. Son necesarias medidas de control y tratamiento de la enfermedad de prevalencia creciente. Objetivo: Evaluar la amplitud de la apertura de la boca en pacientes con disfunción temporomandibular, sometidos a tratamiento con fisioterapia después de 1 y 3 meses de tratamiento. Metodología: Se evaluó la amplitud de la apertura de la boca en 25 pacientes sometidos a tratamiento de fisioterapia. Todos los pacientes fueron diagnosticados con trastorno de acuerdo con el eje 1 del Criterios de diagnóstico de investigación para trastornos temporomandibulares. Los datos recopilados se evaluaron utilizando el programa SPSS y se realizó la prueba de Wilcoxon, con un nivel de confianza del 95\%. Resultados: Para los pacientes que presentaron deterioro de la amplitud máxima de apertura sin asistencia, la fisioterapia permitió una mejora significativa en la ganancia de amplitud en el tiempo inicial y después de un mes de terapia $(p=0,002)$, manteniendo la amplitud hasta el tercer mes, sin mostrar ninguna ganancia significativa. Conclusiones: Para la amplitud variable de apertura de la boca, se muestra que la fisioterapia es una buena alternativa de tratamiento, logrando resultados satisfactorios para aumentar la amplitud y mejorar la condición sintomática del paciente.

Palabras clave: Síndrome de la Disfunción de Articulación Temporomandibular; Articulación Temporomandibular; Fisioterapia; Rango del Movimiento Articular. 


\section{Introdução}

A Disfunção temporomandibular (DTM), termo que abrange alterações articulares e musculares que comprometem a região orofacial ${ }^{1}$, vem sendo um dos distúrbios mais relatados na literatura. Esse distúrbio promove alteração na qualidade de vida dos portadores desta disfunção, uma vez que gera dor e desconforto ${ }^{2}$. Os pacientes diagnosticados com disfunção temporomandibular costumam relatar com grande frequência a presença de dores faciais, sensibilidade muscular e limitação na amplitude de abertura bucal, muitas vezes tendo comprometido os movimentos mandibulares de abertura, fechamento e protrusão ${ }^{2,3,4}$.

Os sintomas desse distúrbio comprometem a qualidade de vida dos pacientes portadores da disfunção, uma vez que causam comprometimento da fala, dores, estalidos, crepitação, dificuldade de alimentação, desvio de trajetória de abertura bucal, entre outras consequências ${ }^{3,4}$. Atualmente já são descritas diversas modalidades de tratamento na literatura para melhora e/ou controle do quadro destes pacientes, tais como: aconselhamento, placa oclusal, acumputura, terapia manual e laserterapia.

A fisioterapia (F) é um conjunto de técnicas de manipulação articular e liberação miofascial de pontos gatilhos ${ }^{5}$. Através da fisioterapia somado ao uso de artifícios como laser, acumputura, exercícios terapêuticos e terapia manual é possível alcançar redução da inflamação dos tecidos envolvidos, promovendo alivio da dor neuromusculoesquelética, reestabelecendo função e devolvendo qualidade de vida ao paciente ${ }^{6,7}$.

Em um estudo foram tratados 30 pacientes com DTM de origem muscular, através de sessões de Fisioterapia ou aplicação de toxina botulínica de forma randomizada. Estes pacientes tiveram acompanhamento de três meses e, ambos os grupos apresentaram melhora significativa no quadro de dor, sem que houvesse diferença significativa entre os grupos ${ }^{8}$. Sendo assim, a Fisioterapia se mostra como uma alternativa de tratamento segura, não invasiva e conservadora para o tratamento de pacientes com DTM.

Este trabalho se propôs a avaliar a amplitude de abertura bucal de pacientes bmetidos ao tratamento com F (fisioterapia) durante o tempo de um a três meses de ompanhamento, buscando descobrir se houve ganho de amplitude de abertura bucal. 


\section{Metodologia}

\section{Natureza do estudo}

Esta pesquisa foi aprovada pelo Comitê de Ética em Pesquisa da Universidade Federal do Rio Grande do Norte (UFRN) antes da sua execução, sob o parecer número 1.442.401. Todos os pacientes foram tratados com fisioterapia $(n=25)$. Todos os pacientes foram diagnosticados com DTM, por um pesquisador capacitado, de acordo com o eixo 1 do RDC/TMD (Research Diagnostic Criteria for Temporomandibular Disorders) versão do formulário publicado por (Pereira et al. (2004) que divide o diagnóstico da disfunção em grupos. O grupo I refere-se a muscular com e sem limitação de abertura, o grupo II refere-se a deslocamento de disco com ou sem redução e/ou limitação de abertura e o grupo III que abrange a artralgia, artrite e artrose. O pesquisador ocupou postura intervencional. Tratou-se de um estudo longitudinal com o tempo de acompanhamento dos pacientes de um (T1) a três meses (T3). A pesquisa foi realizada no Centro Integrado de Atendimento a Portadores de Disfunção do Aparelho Estomatognático (CIADE) que consiste em um Projeto de Extensão do Departamento de Odontologia da UFRN.

\section{Critérios de inclusão e exclusão}

Os pacientes selecionados foram adultos de ambos os gêneros que procuraram o serviço de atendimento do CIADE. Foram incluídos na pesquisa pacientes entre 17 e 50 anos, com diagnóstico positivo para DTM a partir do Eixo I do RDC/TMD, e que referiam sentir dor na região orofacial característica de DTM. Foram excluídos da pesquisa pacientes com comprometimento da capacidade cognitiva que inviabilizasse entendimento das perguntas dos questionários, pacientes com histórico de trauma na cabeça que possa confundir o diagnóstico, pacientes com desordens intracranianas, acientes que fizeram uso de medicação (antiinflamatórios, relaxantes musculares) ra tratamento de DTM nos últimos 3 meses e ainda pacientes que possuem outras usas de dores orofacial, como cáries, doenças periodontais, neuropatia e fibromialgia. Foi realizado o cálculo do poder da amostra com nível de confiança de 
95\% para cada tratamento realizado a fim de detectar a diferença mínima entre os grupos com relação a cada variável.

\section{Instrumento para coleta de dados}

Os dados relacionados à amplitude de abertura bucal foram coletados através do método métrico, com uma régua milimetrada calibrada onde o paciente fazia a abertura bucal máxima com auxílio e era registrada aquela medida. Todos os dados foram coletados nos tempos antes da intervenção com terapia (Baseline $=\mathrm{T} 1)$, um mês após tratamento (T2) e três meses após tratamento intervencional (T3).

\section{Grupo de tratamento}

A fisioterapia seguiu o protocolo ideal, sendo realizada sempre por um mesmo pesquisador capacitado, por oito seções, sendo elas duas vezes por semana com seções de duração de 40 minutos durante 4 semanas. Baseou-se no uso de agentes térmicos (calor e crioterapia), exercícios de alongamento muscular personalizados para cada paciente de acordo com seus sintomas e massagens dos músculos faciais. Os pacientes foram ainda instruídos a realizar em casa, todos os dias, compressas com bolsas de gel mornas $\left(40^{\circ}\right.$ a $\left.50^{\circ}\right)$ por 20 minutos, três vezes ao dia durante todas as 4 semanas de tratamento. As compressas foram aplicadas na região do masseter, temporal e ATM7 .

\section{Análise de dados}

Os dados colhidos foram analisados através do programa SPSS (Statistical Package for the Social Sciences versão 22.0). Para análise do desempenho da terapia ao longo dos três meses de tratamento foi utilizado o teste Wilcoxon, com nível de confiança de $95 \%$. 


\section{Resultados e Discussão}

O número total de pacientes da pesquisa foram 25 participantes, sendo estes $84 \%$ mulheres $(n=21)$ e $16 \%(n=4)$ homens. A idade mínima dos participantes foi de 18 anos e a idade máxima foi 34 anos. Recentemente um estudo buscou descobrir qual perfil mais característico de prevalência de sexo e idade dos pacientes acometidos com DTM, e relatou que $81 \%$ dos pacientes da amostra da pesquisa eram mulheres, com idade média de 36 anos, podendo variar entre 9 e 82 anos $^{9}$. O presente estudo também chegou ao resultado de que mulheres apresentam prevalência maior da doença no serviço no qual a pesquisa foi realizada. A idade média dos participantes desta pesquisa foi de 23 anos, resultado abaixo da idade média reportada na literatura.

Em um cenário em que cada vez mais é crescente o número de indivíduos acometidos com DTM, estudos se fazem cada vez mais necessários para compreender melhor a doença e dessa forma, poder desenvolver estratégias de tratamento eficazes para os pacientes.

Por meio da terapia manual, a fisioterapia atua no tratamento reversível dos sintomas da doença, utilizando exercícios de relaxamento, alongamento e massagens 10. Estudos comprovam que a terapia manual estimula a produção de líquido sinovial e melhora a elasticidade das fibras aderidas ${ }^{11,12}$ explicando dessa forma o ganho na amplitude de abertura bucal. Também existem em outros estudos a vertente que defende que a terapia manual reposiciona a mandíbula no crânio, melhorando sua postura e seu padrão de abertura e consequentemente sua função ${ }^{13}$.

A tabela 1 ilustra os resultados do grupo alcançados na amplitude máxima de abertura sem auxílio. Houve aumento significativo de amplitude entre o T1 e T2 (p = 0.003), porém, não foi observado melhora significativa entre o T1 e T3. $(p=0.008)$. 
Tabela 1: Avaliação da média da amplitude máxima de abertura sem auxílio entre os pacientes tratados com Fisioterapia. Natal, Rio Grande do Norte, Brasil, 2019.

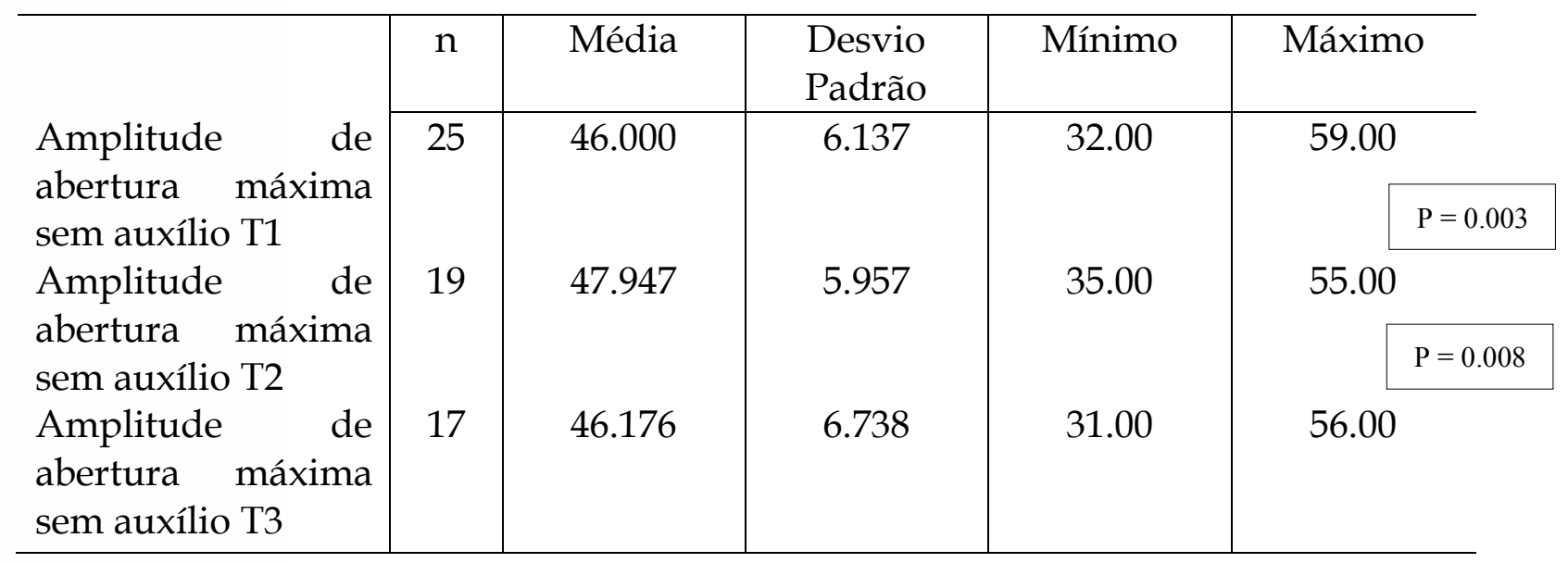

Para o tempo de acompanhamento desse estudo, podemos observar que a fisioterapia apresentou resultados positivos no ganho de amplitude de abertura bucal dos pacientes ao longo do tempo. É importante discutir que a terapia apresentou um melhor desempenho do T1 para o T2, quando houve um ganho real de amplitude de abertura bucal, e quando chegou no T3 o ganho havia se estabilizado, não apresentando grandes variações amplitude de abertura bucal. Uma das razões capazes de explicar esse resultado é que, do T1 para T2 ocorre um choque no organismo uma vez que é realizada uma intervenção, já que está sendo aplicada a terapia. Já do T2 para T3 as estruturas se acostumam com a intervenção e não sofrem mais tanto os efeitos.

Esta pesquisa encontrou resultados que corroboram com aqueles já descritos na literatura. É importante levar em consideração que os pacientes envolvidos nesta pesquisa cínica não apresentavam necessariamente comprometimento de abertura bucal, e sim outras queixas, como dores e estalidos, o que pode justificar de não ter sido tão significativo estatisticamente os resultados obtidos, porém sem dúvidas há melhoras no quadro do paciente, devolvendo conforto e função. Outra limitação deste trabalho é o tempo de acompanhamento reduzido dos pacientes, tendo em vista a importância de acompanhamento a longo prazo dos pacientes tratados. Além disso, pesar do cálculo de poder da amostra dessa pesquisa ter sido de 95\%, sugere-se novos udos com amostras maiores. Devido a escassez de trabalhos na literatura, faz-se 
importante novas pesquisas, considerando reduzida a amostra e o tempo de acompanhamento dos pacientes.

\section{Conclusão}

Para a variável de amplitude de abertura bucal, a estratégia de tratamento através da fisioterapia é capaz de alcançar bons resultados, o que é muito positivo, uma vez que este tratamento é uma terapia reversível e pouco invasiva.

\section{Referências}

1. Okeson, Jeffrey P. Management of temporomandibular disorders and occlusion-E-book. Elsevier Health Sciences, 2014.

2. Ferreira CLP; Silva MMR; Felício CM. Sinais e sintomas de desordem temporomandibular em mulheres e homens. In: CoDAS. Sociedade Brasileira de Fonoaudiologia, CoDAS 2016;28(1):17-21

3. Seabra EJG, Barbosa GAS, Araújo Filho GC, Lima IPC.. Condutas terapêuticas não cirúrgicas relacionadas a DTM's. In: Seabra EJG; Barbosa GAS, Lima IPC. (Org.). Oclusão e DTM: conhecimentos aplicados à clínica odontológica. 1ed.Mossoró: Edições UERN. 2012. 120-137.

4. Greene C. S., Klasser G. D., Epstein, J. B. Revision of the American Association of Dental Research's science information statement about temporomandibular disorders. J Can Dent Assoc, 2010. 76, a115.

5. Kalamir A., Bonello R., Graham P., Vitiello A. L., Pollard H. Intraoral myofascial therapy for chronic myogenous temporomandibular disorder: a randomized controlled trial. Journal of manipulative and physiological therapeutics, 2012. 35(1), 26-37.

6. Rashid A., Matthews N. S., Cowgill H. Physiotherapy in the management of disorders of the temporomandibular joint-perceived effectiveness and access to services: a national United Kingdom survey. British Journal of Oral and Maxillofacial Surgery, 2013. 51(1), 52-57.

7. Michelotti A., Steenks M. H., Farella M., Parisini F., Cimino R., Martina R. The additional value of a home physical therapy regimen versus patient education 
only for the treatment of myofascial pain of the jaw muscles: short-term results of a randomized clinical trial. Journal of orofacial pain, 2004. 18(2), 114-125.

8. Guarda-Nardini L., Stecco A., Stecco C., Masiero S., \& Manfredini, D. Myofascial pain of the jaw muscles: comparison of short-term effectiveness of botulinum toxin injections and fascial manipulation technique. CRANIO®, 2012. 30(2), 95-102.

9. Conti P. C. R., Corrêa A. S. D. M., Lauris J. R. P., Stuginski-Barbosa J. Management of painful temporomandibular joint clicking with different intraoral devices and counseling: a controlled study. Journal of Applied Oral Science, 2015. 23(5), 529-535.

10. Cordeiro I. B., Guimarães A. S. Profile of patients with temporomandibular joint disorder: main complaint, signs, symptoms, gender and age. RGO. Revista Gaúcha de Odontologia (Online), 2012. 60(2), 143-148.

11. Richene R. V., Cordeiro R. S. Atuação Fisioterapeutica Nas Disfunções Da Articulação Temporomandibular. 2019. Journal of Specialist, 3(3):1-21.

12. Basso D., Corrêa E., \& Silva A. M. D. Efeito da reeducação postural global no alinhamento corporal e nas condições clínicas de indivíduos com disfunção temporomandibular associada a desvios posturais. Fisioterapia e Pesquisa, 2010. 17(1), 63-68.

13. Gomes N. C., Berni-Schwarzenbeck K., Packer A. C., Rdrigues-Bigaton D. Efeito da estimulação elétrica de alta voltagem catódica sobre a dor em mulheres com DTM. Brazilian Journal of Physical Therapy, 2012. 16(1), 10-15. 